\title{
LAPTM4B promotes the progression of bladder cancer by stimulating cell proliferation and invasion
}

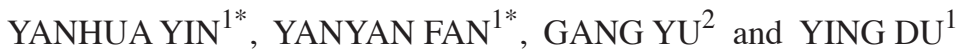 \\ ${ }^{1}$ Department of Pathology, Liaocheng People's Hospital; ${ }^{2}$ Department of Public Health, \\ Liaocheng Cancer Hospital, Liaocheng, Shandong 252000, P.R. China
}

Received April 28, 2021; Accepted July 29, 2021

DOI: 10.3892/ol.2021.13026

\begin{abstract}
Bladder cancer is a highly metastatic tumor and one of the most common malignant tumors originating in the urinary system. Due to the complicated etiology and lack of significant early symptoms, the diagnosis and treatment of bladder cancer is difficult. Lysosome-associated transmembrane protein $4 \beta$ (LAPTM4B) was reported to be involved in the development and progression of several types of tumor, however, its potential effect on the development and metastasis of bladder cancer is still unclear. Immunohistochemistry was performed to detect the protein expression level of LAPTM4B in bladder cancer tissues and short hairpin RNAs targeting LAPTM4B were transfected into bladder cancer cells to knockdown its expression. MTT and colony formation assays were performed to detect cell proliferation, while wound healing and Transwell invasion assays were performed to detect cell migration and invasion, respectively. In addition, tumor growth assays were performed to confirm the effects of LAPTM4B in mice. The present study demonstrated that LAPTM4B was associated with the prognosis of patients with bladder cancer. In addition, LAPTM4B was associated with clinical characteristics, including tumor stage and recurrence. The results further showed that LAPTM4B knockdown could suppress the proliferation of bladder cancer cell lines. In addition, the migration and invasion of T24 and 5637 cells was suppressed following LAPTM4B knockdown in vitro. The in vivo data confirmed that knockdown of LAPTM4B markedly inhibited tumor growth and metastasis in mice. In summary, the results from the present study provide strong evidence of the effects of LAPTM4B in bladder cancer progression.
\end{abstract}

Correspondence to: Professor Ying Du, Department of Pathology, Liaocheng People's Hospital, 67 Dongchang West Road, Liaocheng, Shandong 252000, P.R. China

E-mail: lucysandi18@163.com

*Contributed equally

Key words: bladder cancer, LAPTM4B, proliferation, invasion, T24

\section{Introduction}

Bladder cancer is a highly metastatic tumor $(1,2)$. It is also one of the most common malignant tumors originates in the urinary system (3). The morbidity rate of bladder cancer is second only to prostate cancer in Western countries, and in 2018, there were $~ 549,400$ new cases and 200,000 deaths (4). However, no significant symptoms of bladder cancer at the early stage makes early diagnosis and treatment more difficult (5). Targeted therapy for bladder cancer has become an effective treatment method $(6,7)$. Therapeutic targets for bladder cancer have already made some progress; however, it is still hard to meet the therapeutic requirement $(8,9)$. Thus, novel and promising therapeutic targets are still required.

Lysosome-associated transmembrane protein $4 \beta$ (LAPTM4B) localizes on the late lysosomes by its lysosome localization motif. It could affect several cellular functions by regulating multiple signaling pathways $(10,11)$. LAPTM4B is involved in the process of autophagy (12). In addition, LAPTM4B could contribute to the recruitment of the amino acid transporter to lysosomes; therefore, promoting the uptake of lysosomal leucine (13). Mutations in LAPTM4B could lead to serious genetic diseases, such as Myocardial Ischemia/Reperfusion Injury (14).

LAPTM4B was firstly found to be highly expressed in hepatocellular carcinoma (HCC), and has been associated with poor prognosis in patients with HCC, breast cancer, gastric cancer, and acute myeloid leukemia (15-18). LAPTM4B was also associated with the susceptibility to non-small cell lung and ovarian cancers (18). In the development of HCC, LAPTM4B and AP4 play synergistic roles (19). LAPTM4B could also promote the development of gastric cancer via EGFR over-activation, which was repressed by Beclin1 (20). Collectively, LAPTM4B could promote the proliferation and invasion of cancer cells, induce autophagy, apoptosis, and assist drug resistance (20). LAPTM4B affects the pathogenesis of multiple types of tumor; however, its potential role in bladder cancer progression remains unknown.

In the present study, it was found that LAPTM4B was associated with the poor prognosis of patients with bladder cancer. LAPTM4B knockdown notably inhibited cell proliferation, migration and invasion, and suppressed tumor growth and metastasis in mice. Thus, LAPTM4B could affect the progression of bladder cancer in vitro and in vivo. 


\section{Materials and methods}

Antibodies, primers and short hairpin (sh) RNA plasmids. The anti-LAPTM4B rabbit polyclonal [for immunohistochemistry (IHC), 1:100 dilution; for western blot analysis, 1:1,000 dilution; cat. no. PA5-43047] antibody was purchased from Thermo Fisher Scientific, Inc., and the anti- $\beta$-actin mouse monoclonal (1:1,000 dilution; cat. no. ab8226) was purchased from Abcam.

The following primers were used: LAPTM4B forward, 5'-GGAACTGCTACCGATACATCAA-3' and reverse, 5'-TCA CAGTGGCATCATCATACG-3'; $\beta$-actin forward, 5'-CAG CTCACCATGGATGATGATATC-3' and reverse, 5'-AAG CCGGCCTTGCACAT-3'.

The pLVX shRNA plasmid of LAPTM4B (cat. no. 101111) was purchased from OriGene Technologies, Inc., and has the following targeted sequence: 5'-GGTCGCCTTCGGAGC GAAGGGTA-3'.

Human tissue samples and analysis. A total of 111 human bladder tumor tissues were collected from patients (mean age 67.5; 71 males and 40 females) with bladder cancer, and adjacent tissues ( $5 \mathrm{~mm}$ from the tumor tissues) following surgery at Liaocheng People's Hospital (Shandong, China) between September 2015 and June 2018. The clinicopathological features, including age, sex, tumor stage, tumor grade, lymph node metastasis and recurrence (tumor redevelops at the original site) were respectively recorded and analyzed at Liaocheng People's Hospital.

To further investigate the association between LAPTM4B expression level and bladder cancer, IHC was performed. In brief, the samples were fixed with $10 \%$ formalin for $24 \mathrm{~h}$ at $98^{\circ} \mathrm{C}$, embedded with resin (Epoxy resin; Sigma-Aldrich; Merck $\mathrm{KGaA}$ ), and divided into $5-\mu \mathrm{m}$ thick sections. The sections were dewaxed with xylene at $65^{\circ} \mathrm{C}$, then rehydrated in a gradient ethanol series. The samples were immersed in citrate buffer $(\mathrm{pH}, 6.0)$ at $98^{\circ} \mathrm{C}$ for $30 \mathrm{~min}$ and placed in a microwave for incubation for $10 \mathrm{~min}$ for antigen retrieval. Then, hydrogen peroxide was added to block endogenous peroxidase activity and the samples were incubated at room temperature for $10 \mathrm{~min}$, followed by blocking with $2 \% \mathrm{BSA}$ (Sigma-Aldrich; Merck KGaA) for 20 min at room temperature. Subsequently, the samples were incubated with the LAPTM4B antibody at room temperature for $2 \mathrm{~h}$. Lastly, the samples were washed with PBS, 4 times and incubated with the secondary antibody (rabbit; 1:200 dilution; cat. no. ab205718; Abcam). Diaminobenzidine was used as a chromogen substrate. Images were captured using an Olympus inverted fluorescence microscope (IX71; Zeiss AG).

The proportion of positive stained cells was scored as follows: 0 , negative; $1,10-50 \%$ positive and $2,>50 \%$ positive staining. The staining intensity was assessed on a score of 0 (negative level staining), 1 (low level staining), and 2 (high level staining). The expression levels of LAPTM4B were further examined based on a staining index: Staining intensity score plus staining percentage score. Staining index, 0-2 indicated low expression, while 3/4 indicated high expression. The Kaplan-Meier survival analysis of overall survival (OS) and progression-free survival (PFS) rates was performed between LAPTM4B low and high expression groups. OS was defined as the time from recruitment into the study to death from any cause. PFS was defined as the period after treatment when the disease was stable and did not progress. The development of the disease was followed up every 6 months.

Cell culture and transfection. The T24 and 5637 bladder tumor cell lines were purchased from American Type Culture Collection. Both the cell lines were maintained in RPMI-1640 medium, supplemented with 10\% FBS (Gibco; Thermo Fisher Scientific, Inc.) and incubated at $37^{\circ} \mathrm{C}$ in a humidified incubator with $5 \% \mathrm{CO}_{2}$.

The shRNA plasmids of LAPTM4B were transfected into the bladdercancercell lines using Lipofectamine ${ }^{\circledR} 2000$ (Invitrogen; Thermo Fisher Scientific, Inc.) at $37^{\circ} \mathrm{C}$ for $4 \mathrm{~h}$. The scrambled control plasmid (5'-ATGGTACTGACCTCCAGAG-3') was used as the negative control (NC). A total of $1 \times 10^{5}$ cells per well were seeded into 6-well plates and a total of 2 groups were used: sh-LAPTM4B group $(0.5 \mu \mathrm{g})$ and sh-NC group $(0.5 \mu \mathrm{g})$. Knockdown efficiency was measured by both reverse transcription-quantitative PCR (RT-qPCR) and western blot analysis after $48 \mathrm{~h}$. These cells were used to assess the association between LAPTM4B expression level and cellular processes. Subsequently the cells in which LAPTM4B was stably knocked down were screened and used for the in vitro and in vivo assays.

$R T$ - $q P C R$. Total RNA was isolated from the T24 and 5637 cell lines or tumor tissues using TRIzol ${ }^{\circledR}$ (Invitrogen; Thermo Fisher Scientific, Inc.). Subsequently, the total RNA was reverse transcribed using $\mathrm{M}-\mathrm{MLV}$ reverse transcriptase at $42^{\circ} \mathrm{C}$ for $60 \mathrm{~min}$ (Promega Corporation). qPCR was conducted using SYBR PrimeScript RT-PCR kit II (cat. no. DRR083; Takara Biotechnology Co., Ltd.) and the relative expression levels of LAPTM4B was normalized to the mRNA expression levels of $\beta$-actin. The following thermocycling conditions were used: Initial denaturation at $95^{\circ} \mathrm{C}$ for $3 \mathrm{~min}$; followed by 30 cycles of denaturation at $95^{\circ} \mathrm{C}$ for $30 \mathrm{sec}$, annealing at $58^{\circ} \mathrm{C}$ for $30 \mathrm{sec}$ and extension at $72^{\circ} \mathrm{C}$ for $30 \mathrm{sec}$. The $2^{-\Delta \Delta \mathrm{Cq}}$ method was used to quantify the results (21).

Western blot analysis. The bladder cancer cells or tissues were lyzed with lysis buffer [60 mM Tris- $\mathrm{HCl}(\mathrm{pH} 6.8)$, $2 \%$ SDS, $20 \%$ glycerol, $0.25 \%$ bromophenol blue, $1.25 \%$ 2-mercaptoethanol and protease inhibitor cocktail]. Protein determination was performed using the BCA method. A total of $10 \mu \mathrm{g}$ of each protein sample was loaded per lane and separated using 10\% SDS-PAGE. Then, the proteins were transferred onto PVDF membranes (cat. no. IPSN07852; MilliporeSigma) and the membranes were blocked with $5 \%$ skimmed milk in TBS-Tween-20 (0.5\%) buffer and subsequently incubated with the primary antibodies for $2 \mathrm{~h}$ at room temperature. Following which, the membranes were incubated with the secondary antibody (rabbit; 1:5,000 dilution; cat. no. ab205718; Abcam) for $45 \mathrm{~min}$ at room temperature. Each blot was subsequently visualized with an ECL kit (cat. no. RPN 2109; GE Healthcare).

Colony formation assay. Approximately 1,000 bladder cancer cells were added into 6-well plates and cultured at $37^{\circ} \mathrm{C}$ for $48 \mathrm{~h}$ after transfection. After 14 days, the cells were 
subsequently fixed with $4 \%$ paraformaldehyde for $30 \mathrm{~min}$ at room temperature and stained with $0.2 \%$ crystal violet at room temperature for $20 \mathrm{~min}$, then washed with PBS for 4 times. Colony numbers were manually counted using an Olympus inverted fluorescence microscope and images captured (IX71; Zeiss AG). A colony was counted when it included $>100$. The number of colonies per visual field area visible under the microscope was counted.

MTT assay. The cells, transfected with shRNA plasmids, were added into 96-well plates for 3 days. Subsequently, the cells were treated with MTT for $3 \mathrm{~h}$, then $200 \mu \mathrm{l}$ dimethyl sulfoxide was added to dissolve the purple formazan and the OD value was measured using a microplate reader at $570 \mathrm{~nm}(22)$.

Transwell invasion assay. The T24 and 5637 cells, transfected with shRNA plasmid, were subsequently used for Transwell invasion assays. The upper chambers were coated with $20 \%$ Matrigel in RPMI-1640 medium and incubated at $37^{\circ} \mathrm{C}$ for $1 \mathrm{~h}$. A total of $1 \times 10^{5}$ cells in $150 \mu \mathrm{l}$ serum-free medium was then added into the upper chambers of the inserts. Medium with $10 \%$ FBS was added into the bottom chamber to stimulate cell invasion. After $24 \mathrm{~h}$ incubation at $37^{\circ} \mathrm{C}$, the cells were fixed with $4 \%$ paraformaldehyde for $25 \mathrm{~min}$ at room temperature and stained with $0.2 \%$ crystal violet for $15 \mathrm{~min}$ at room temperature. Then, images were captured and the number of invaded cells were counted using an Olympus inverted fluorescence microscope (IX71; Zeiss AG).

Wound healing assay. Both the T24 and 5637 cell lines were transfected with shRNA plasmids and cultured to form confluent monolayers. After the confluence reached $100 \%$, the wound was created using a $20 \mu$ l pipette tip. Cell debris was washed with PBS 3 times. The serum-free culture medium was added to the cells and the cells were cultured. Images of the wounds were captured at 0 and $24 \mathrm{~h}$, and the extent of healing was measured. Migration ability was measured using ImageJ software (v1.8.0; National Institutes of Health) and quantified as a percentage of wound width (post-healing wound width/pre-healing wound width).

Tumor growth and lung metastasis analysis. All animal procedures were approved by the Institutional Animal Care and Use Committee of Liaocheng People's Hospital (Shandong, China). A total of 12 male nude BalB/c mice (8-weeks-old; 18-22 g) were purchased from Beijing Vital River Laboratory Animal Technology Co., Ltd., and fed with food and water ad libitum and at specific pathogen-free conditions $\left(20^{\circ} \mathrm{C} ; 60 \%\right.$ humidity and alternating 12-h light/dark cycles).

For the tumor growth assay, the T24 cell line was stably transfected with shRNA plasmids, then $3 \times 10^{6}$ cells were inoculated into the Balb/c nude mice and tumors formed 2 weeks later. The tumor growth curves were analyzed 7 weeks later. The tumor size was calculated using the following formula: Tumor volume=length $\mathrm{x}$ width $\mathrm{x}$ width $/ 2$. The mice were euthanized with an intraperitoneal injection of $120 \mathrm{mg} / \mathrm{kg}$ sodium pentobarbital before the tumor was removed. The hearts of the mice were then monitored and death was confirmed by cardiac arrest. There were six mice in the control and LAPTM4B knockdown group.
To further detect the expression level of the LAPTM4B in the tumor tissues from the mice, IHC was performed as aforementioned. Subsequently, the staining intensity of LAPTM4B in the tumor tissues was measured using ImageJ software (v1.8.0; National Institutes of Health) and analyzed statistically using the staining intensity, which was presented as the median \pm interquartile range, including the Q1/Q3 quartiles.

For the lung metastasis assay, $1 \times 10^{6} \mathrm{~T} 24$ cells, transfected with shRNA plasmids, were resuspended in $150 \mu$ l PBS buffer and injected into the tail vein to stimulate lung metastasis. After 7 weeks, all the mice were sacrificed and the lungs were isolated and images were captured, and the metastasis degree was measured.

Statistical analysis. GraphPad v6.0 software (GraphPad Software, Inc.) was used for statistical analysis. The in vitro and in vivo results were presented as the mean \pm standard deviation. The analysis between clinical features and LAPTM4B expression was performed using a $\chi^{2}$ test. A Student's t-test was used for statistical comparisons. The survival rates between cancer progression and LAPTM4B expression was analyzed using the Kaplan-Meier method and the log-rank test. IHC staining intensity in animal experiments was presented as the median + interquartile range and analyzed using Mann-Whitney U test. Each experiment was repeated 3 times. $\mathrm{P}<0.05$ was considered to indicate a statistically significant difference.

\section{Results}

Expression of LAPTM4B was associated with the prognosis and clinical features of patients with bladder cancer. To investigate the potential role of LAPTM4B in bladder cancer progression, IHC, clinicopathological characteristic analysis and Kaplan-Meier analysis were all conducted. The expression level of LAPTM4B in human tumor tissues from patients with bladder cancer, who underwent surgical resection was detected using IHC. According to the IHC staining results, LAPTM4B was mainly expressed in the cytoplasm and membrane of human bladder cancer tissues (Fig. 1A). A total number of 111 surgical samples were classified into LAPTM4B low and high-expression groups according to the staining intensity (Fig. 1A). In comparison, corresponding non-tumor tissues showed low expression level of LAPTM4B (Fig. 1B). Based on the expression level in bladder tumor tissues, 34 patients showed low LAPTM4B expression, whereas 77 showed high expression (Table I).

The clinicopathological characteristics of patients with bladder cancer were analyzed between the LAPTM4B low and high expression level groups. Patient age, sex, tumor size, grade and lymph node metastasis, were recorded and analyzed. Based on the analysis results, no significant difference was found in these features between the LAPTM4B low and high expression groups (Table I). Notably, LAPTM4B expression level in the bladder tumor tissues was significantly associated with the tumor stage $(\mathrm{P}=0.004)$ and recurrence $(\mathrm{P}=0.014)$.

Kaplan-Meier survival analysis was used to investigate the association between LAPTM4B and the prognosis of patients with bladder cancer. Data showed that LAPTM4B expression 
Table I. Association between LAPTM4B and the clinicopathological characteristics in 111 patients with bladder cancer.

\begin{tabular}{|c|c|c|c|c|c|}
\hline \multirow[b]{2}{*}{ Characteristic } & \multirow[b]{2}{*}{ Total number } & \multicolumn{2}{|c|}{ LAPTM4B expression } & \multirow[b]{2}{*}{$\chi^{2}$} & \multirow[b]{2}{*}{ P-value } \\
\hline & & $\begin{array}{l}\text { Low } \\
\mathrm{n}=34\end{array}$ & $\begin{array}{l}\text { High } \\
\mathrm{n}=77\end{array}$ & & \\
\hline Age, years & & & & 2.199 & 0.138 \\
\hline$<55$ & 44 & 17 & 27 & & \\
\hline$\geq 55$ & 67 & 17 & 50 & & \\
\hline Sex & & & & 0.933 & 0.334 \\
\hline Male & 71 & 24 & 47 & & \\
\hline Female & 40 & 10 & 30 & & \\
\hline Tumor stage & & & & 8.516 & 0.004 \\
\hline $\mathrm{T} 2$ & 52 & 23 & 29 & & \\
\hline $\mathrm{T} 3 / \mathrm{T} 4$ & 59 & 11 & 48 & & \\
\hline Tumor grade & & & & 1.321 & 0.250 \\
\hline Low & 31 & 12 & 19 & & \\
\hline High & 80 & 22 & 58 & & \\
\hline Lymph node metastasis & & & & 3.074 & 0.080 \\
\hline Yes & 33 & 14 & 19 & & \\
\hline No & 78 & 20 & 58 & & \\
\hline Recurrence & & & & 5.983 & 0.014 \\
\hline Yes & 52 & 10 & 42 & & \\
\hline No & 59 & 24 & 35 & & \\
\hline
\end{tabular}

LAPTM4B, lysosome-associated transmembrane protein $4 \beta$.

A
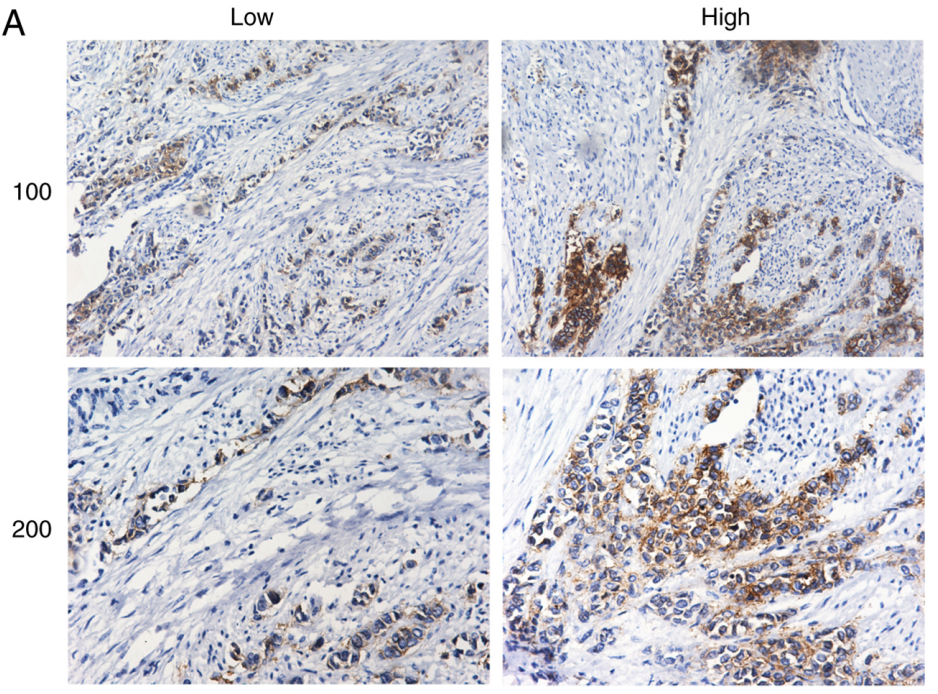

B

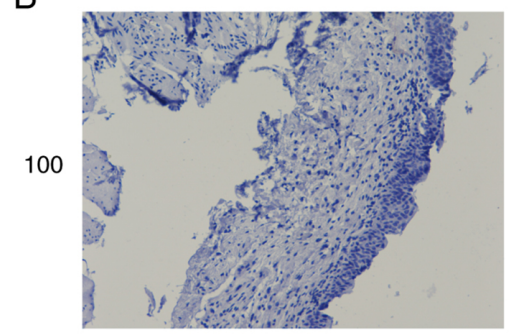

200

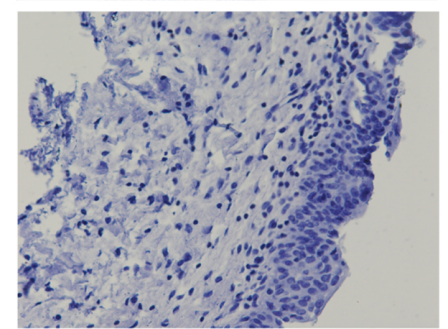

- LAPTM4B low

- LAPTM4B high
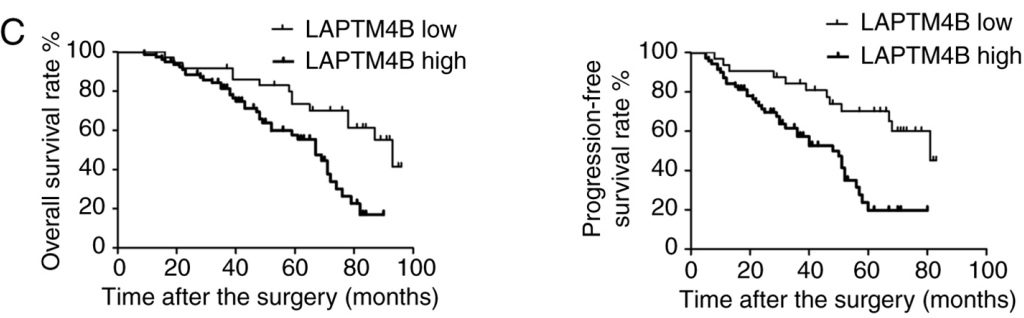

Figure 1. LAPTM4B is associated with the prognosis of patients with bladder cancer. (A) IHC was performed and representative images of LAPTM4B expression in bladder tumor tissues are shown. Magnification, x100 and x200. (B) IHC showed the expression level of LAPTM4B in the adjacent non-tumor tissues. Magnification, x100 and x200. (C) The Kaplan-Meier survival analysis of overall survival and progression-free survival rates between LAPTM4B low and high expression groups. IHC, immunohistochemistry; LAPTM4B, lysosome-associated transmembrane protein $4 \beta$. 
A

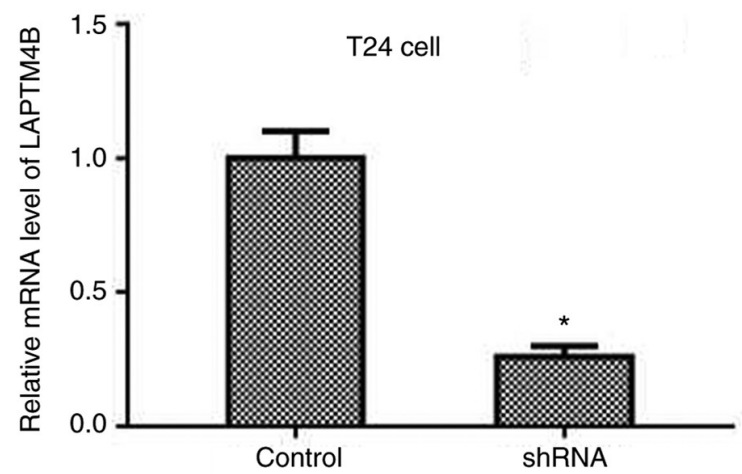

B

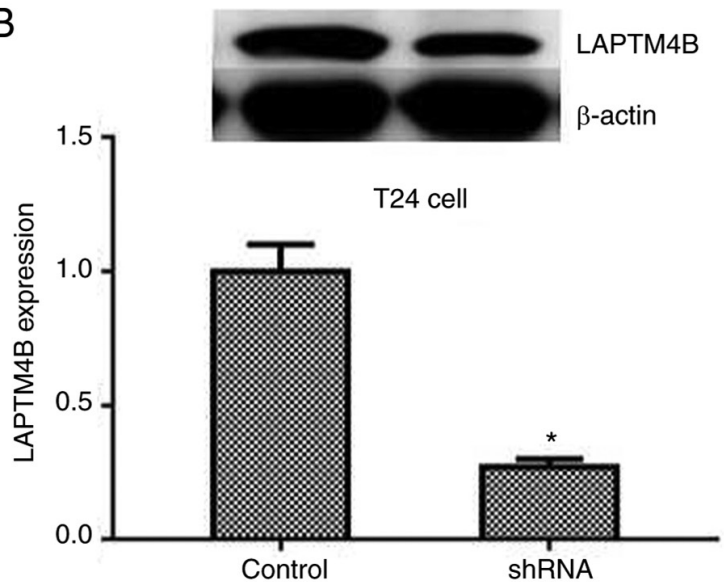

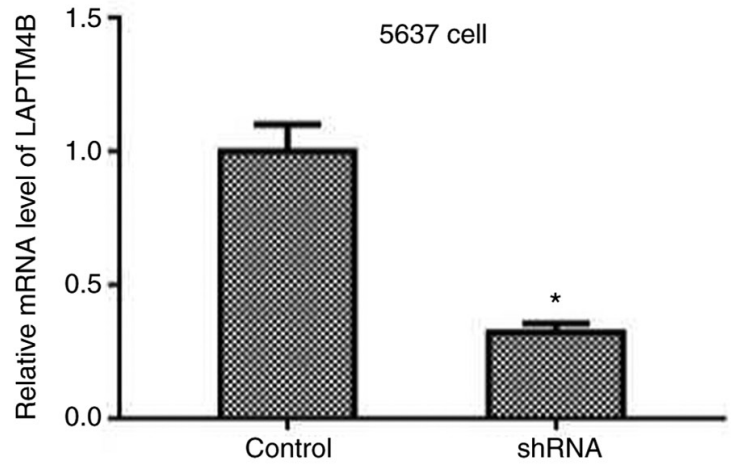

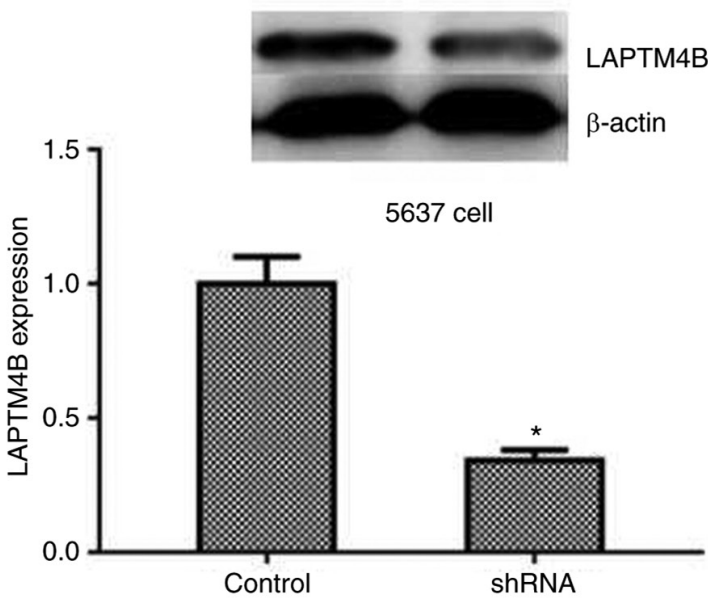

Figure 2. LAPTM4B expression was effectively knocked down in both the T24 and 5637 human bladder cancer cell lines from the transfection of LAPTM4B shRNA. (A) Quantitative PCR and (B) western blot analysis revealed the significantly reduced mRNA and protein expression levels of LAPTM4B following transfection with target shRNA in the T24 and 5637 cell lines, respectively. The results are presented as the mean $\pm \mathrm{SD}$. $\mathrm{P}<0.05$. LAPTM4B, lysosome-associated transmembrane protein $4 \beta$; sh, short hairpin.

was associated with OS and PFS rates (Fig. 1C). These results indicated that LAPTM4B was associated with poor prognosis in patients with bladder cancer.

LAPTMAB was associated with bladder cancer cell proliferation and invasion in vitro. To further investigate the mechanism of LAPTM4B regulation in the progression of bladder cancer, a shRNA targeting LAPTM4B was transfected into the two types of bladder cancer cell lines, T24 and 5637, to suppress the expression of LAPTM4B. qPCR (Fig. 2A) and western blot analysis (Fig. 2B) suggested that transfection with LAPTM4B shRNA effectively knocked down LAPTM4B expression in the T24 and 5637 cell lines.

To investigate the potential effect of LAPTM4B on the proliferation of bladder cancer, colony formation assays were conducted. It was found that LAPTM4B knockdown markedly inhibited the proliferation of the T24 and 5637 cell lines, from the decrease in cell colony numbers (Fig. 3A). Similarly, there was lower proliferation from the MTT assay, as shown from the lower OD values in Fig. 3B.

The effects of LAPTM4B on the migration and invasion of bladder cancer cells were also investigated. As expected, knockdown of LAPTM4B also slowed down the extent of wound closure in the two bladder cancer cell lines (Fig. 3C). In addition, T24 and 5637 cells exhibited a significantly lower invasive ability following LAPTM4B knockdown, with markedly decreased the number of invasive cell (Fig. 3D).
Taken together, it was found that LAPTM4B was associated with the regulation of bladder cancer cell proliferation, and migration and invasion in vitro.

LAPTM4B promotes bladder tumor growth and metastasis in mice. According to the in vitro findings, knockdown of LAPTM4B reduced the proliferation, and migration and invasion of bladder cancer cell lines; therefore, the role of LAPTM4B in the growth and metastasis of bladder cancer was investigated further in mice.

The T24 cell lines, transfected with control or LAPTM4B shRNA plasmids, were inoculated into Balb/c nude mice and tumor formation began 2 weeks later. The notable low mRNA expression levels of LAPTM4B in the knockdown group (Fig. 4A), confirmed successful transfection. Representative images of the tumors were captured and are shown in Fig. 4B. The maximum volume of tumor in mice was $10 \mathrm{~mm}$. According to the results, the volume of the tumors from the LAPTM4B knockdown mice was significantly smaller compared with that in the control mice (Fig. 4B). Furthermore, lung metastasis assay was performed in the mice and the volume of lung metastasis in the mice with LAPTM4B-knockout T24 cells was markedly decreased compared with that in the mice transfected with the control shRNA (Fig. 4C).

IHC further confirmed the knockdown of LAPTM4B expression in the tumor tissues (Fig. 4D). Therefore, all these 

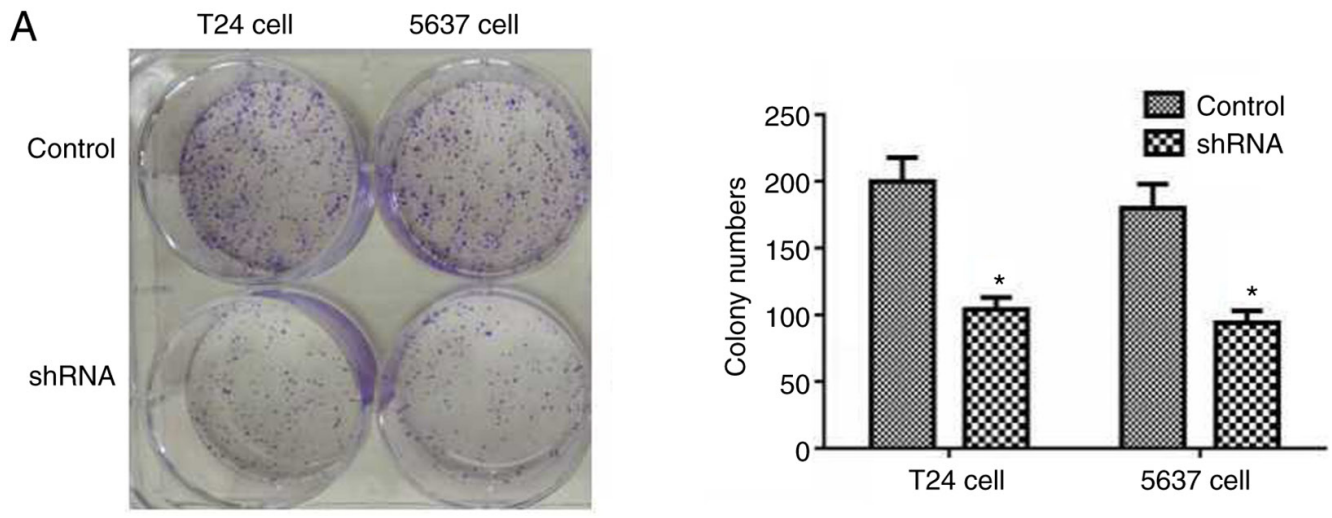

B

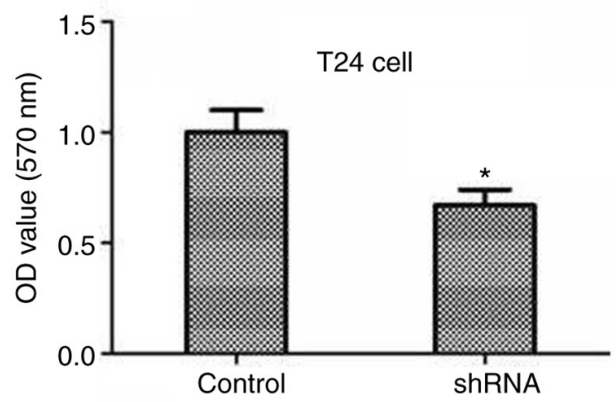

C

T24 cells

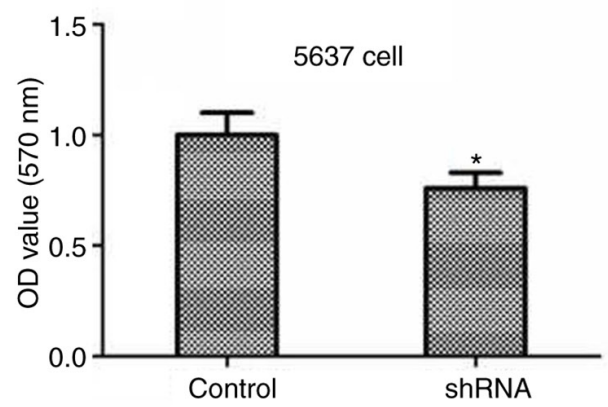

5637 cells

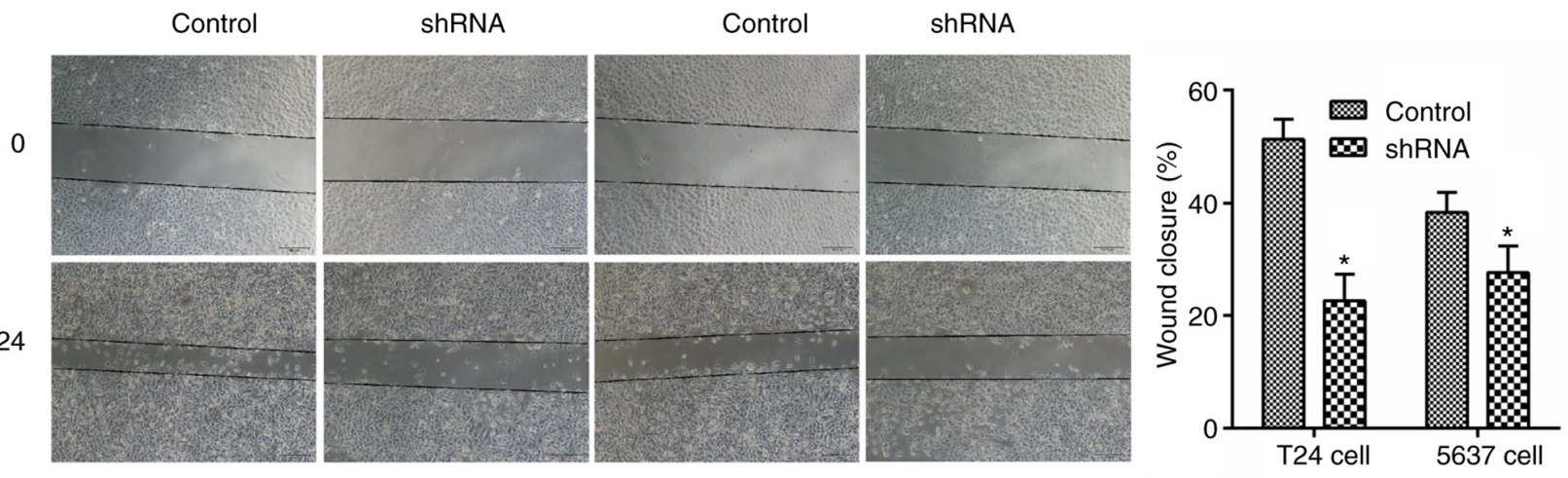

D

T24 cells

5637 cells

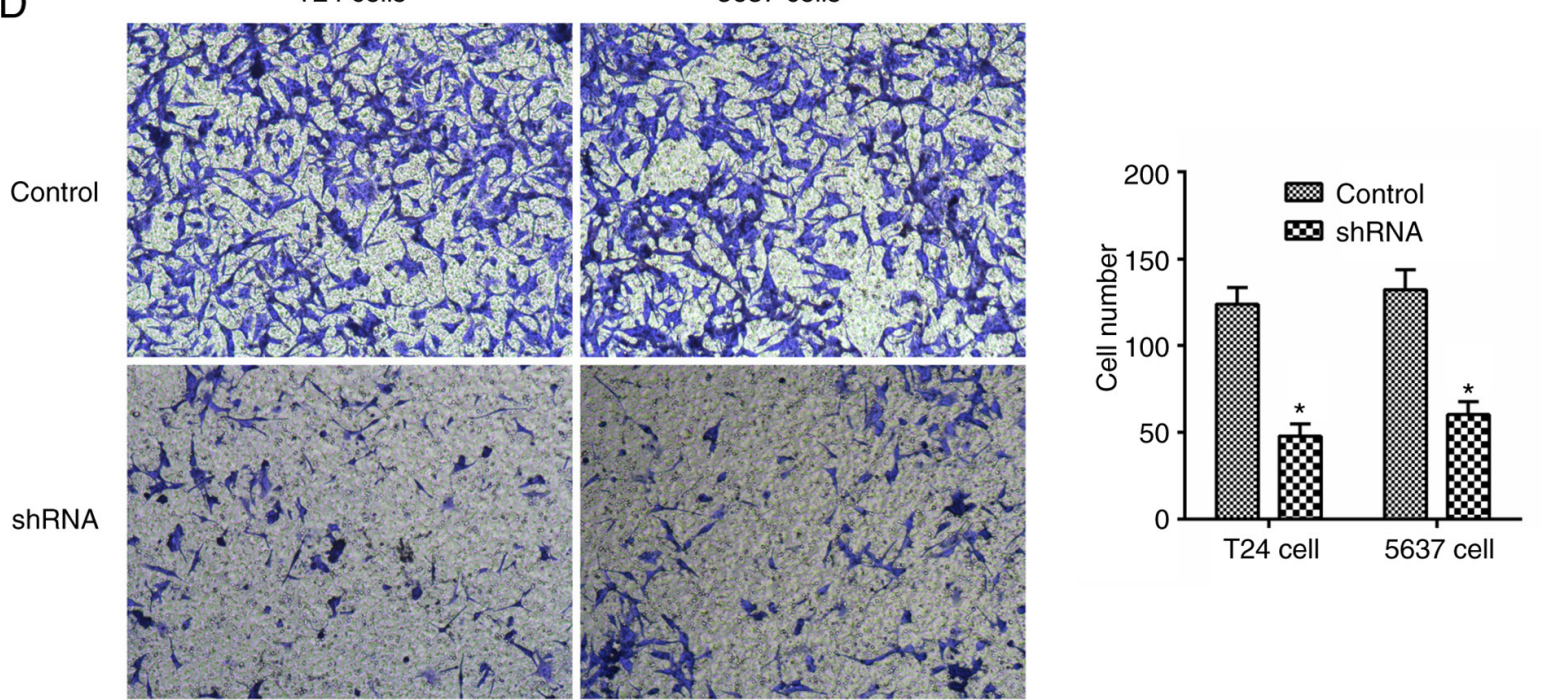

Figure 3. LAPTM4B promotes the proliferation, and migration and invasion of bladder cancer cells in vitro. (A) Representative images of colony formation assays in the T24 and 5637 cell lines transfected with control or LAPTM4B shRNA. (B) The results of MTT assays showed the inhibition of cell proliferation caused by knockdown of LAPTM4B. (C) Wound healing assays were performed in the T24 and 5637 cells transfected with control or LAPTM4B-shRNA and images were captured at 0 and $24 \mathrm{~h}$ time points. (D) Transwell invasion assays were performed in cells transfected with siRNA control or LAPTM4B in the T24 and 5637 cell lines and the number of invasive cells were quantified and analyzed statistically. The results are presented as the mean \pm SD. ${ }^{*} \mathrm{P}<0.05$. OD, optical density; sh, short hairpin. 
A

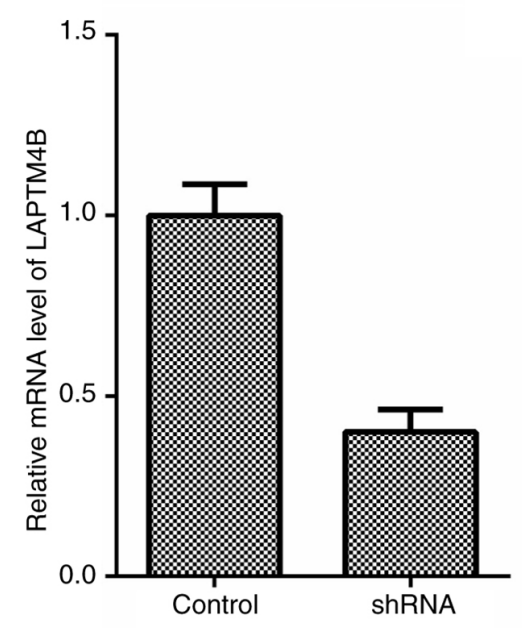

C

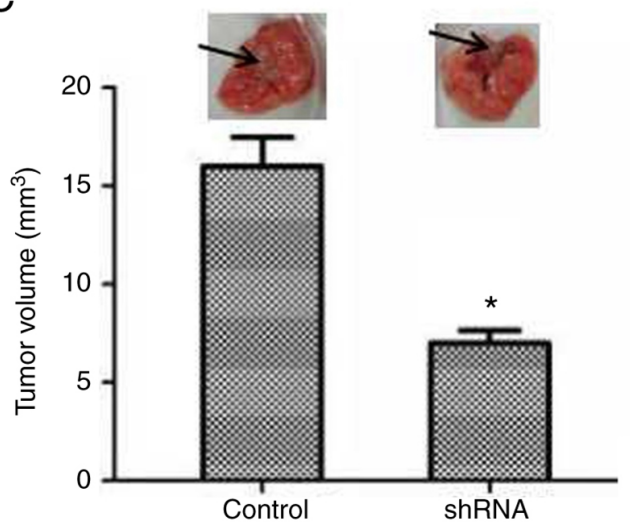

$\mathrm{B}$
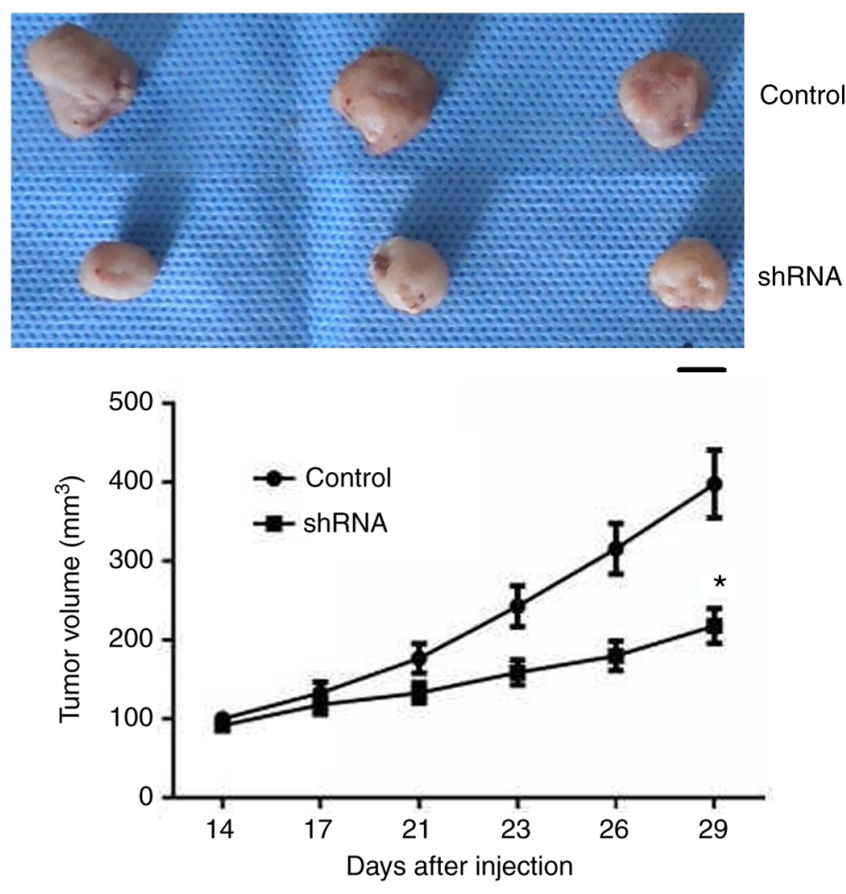

D
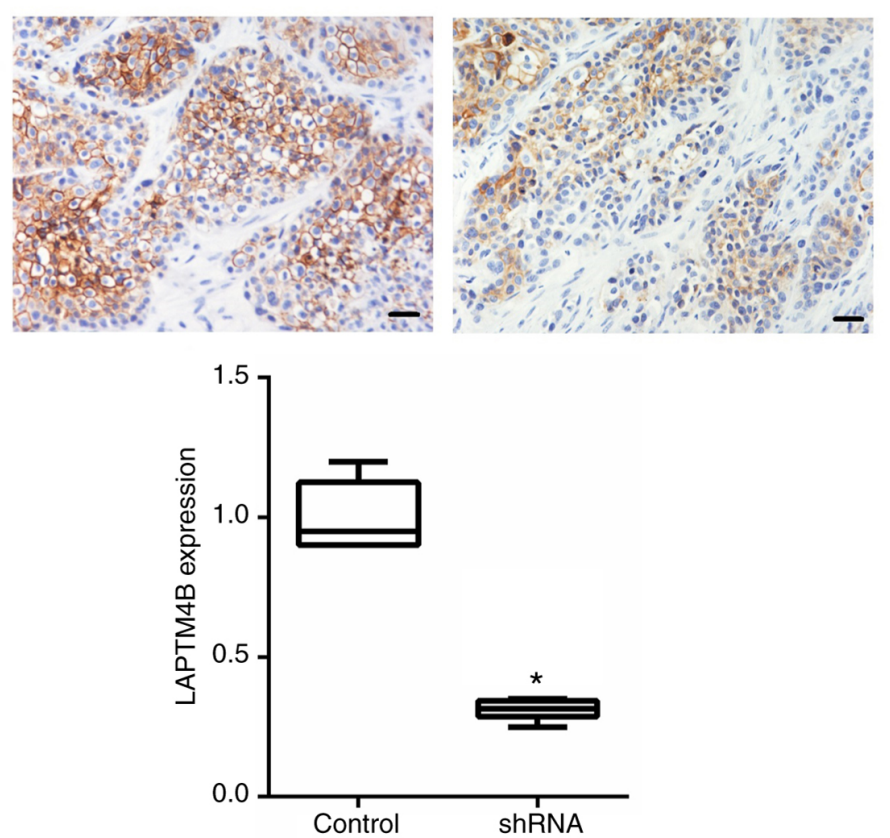

Figure 4. LAPTM4B knockdown impairs bladder cancer growth and development in vivo. T24 cells transfected with LAPTM4B or control shRNA plasmids were implanted into nude mice. After 2 weeks, the tumors were isolated and the volume was calculated each week. $n=6$ in each group. (A) The mRNA expression levels of LAPTM4B in control and LAPTM4B knockdown tumors. (B) Tumor images are shown and the growth curves were calculated based on the average volume of 6 tumors in each group. Scale bar, $5 \mathrm{~mm}$. (C) Lung metastasis was performed and the representative images of the lungs in the control or LAPTM4B shRNA groups are shown. (D) Immunohistochemistry was used to determine the expression level of LAPTM4B and the staining intensity in the control or LAPTM4B knockdown tumor tissues, isolated from mice was determined. Scale bar, $0.5 \mathrm{~mm}$. The results are presented as the mean \pm SD in (A-C) and mean \pm range in (D). " $\mathrm{P}<0.05$. LAPTM4B, lysosome-associated transmembrane protein $4 \beta$; sh, short hairpin.

data confirmed that LAPTM4B was associated with the regulation of bladder cancer growth and development in vivo.

\section{Discussion}

Over the past few decades, the mortality rate of bladder cancer has increased (23). With the clinical application of new treatment, the treatment effect of bladder cancer has been significantly improved (3). However, targeted therapy is undoubtedly the most promising choice, and highly effective therapeutic targets for bladder cancer is still required $(6,24)$. Some gene mutations, such as in osteopontin and CTLA4, result in the development of bladder cancer, which can be used as potential targets for treatment (25). In addition, a variety of new therapeutic targets, such as proteins or long non-coding RNA, have been discovered, which provide significant convenience for the study of tumor mechanism and treatment (26-28). In the present study, LAPTM4B, a regulator 
of multiple types of tumor (10-12,29,30), was associated with the progression of bladder cancer. LAPTM4B has the potential to be a novel therapeutic target for the treatment of bladder cancer; however, the molecular mechanisms require further investigation.

LAPTM4B was also associated with some of the clinical features, including tumor stage and recurrence, further suggesting that LAPTM4B might promote poor prognosis and distant metastasis of bladder cancer, which is consistent with the results from the in vitro experiments, that LAPTM4B promoted bladder cancer proliferation, and migration and invasion. Further clinical studies and experiments are required to investigate how LAPTM4B precisely regulates the pathogenesis of bladder cancer.

LAPTM4B, as a transmembrane protein, has been reported to be associated with poor prognosis in multiple types of cancer, whereas its precise physiological function is not well understood (10-12,29-31). Previous studies confirmed that LAPTM4B could interact with ceramide to promote its removal from late endosomal organelles; therefore, regulating key sphingolipid-mediated cell death processes $(10,12,29)$. A previous study also indicated that LAPTM4B was critical for autophagic maturation (32). The overexpression of LAPTM4B promoted autophagy, which led to cancer cell proliferation (12). It was found that LAPTM4B knockdown inhibited the proliferation of bladder cancer cells; however, the association between LAPTM4B and autophagy also requires further investigation. In addition, it was reported that LAPTM4B, in cooperation with AP4, activated the PI3K/AKT signaling pathway and the caspase-dependent pathway to promote the proliferation and invasion of HCC (32). Notably, it was found that knockdown of LAPTM4B significantly blocked proliferation and invasion of bladder cancer in vitro and in mice, which might be partly caused by the activation of these pathways.

In addition to the association between LAPTM4B and bladder cancer found in the present study, LAPTM4B has been associated with the growth and metastasis of several types of tumor. Firstly, LAPTM4B knockdown markedly blocked the proliferation and invasion of HeLa cells in vitro (33), which is consistent with the present study. LAPTM4B activated the EGFR signaling pathway and further promoted the development of gastric cancer, which was repressed by Beclin1 (20). A report demonstrated that overexpression of LAPTM4B induced cell proliferation, migration, and simultaneous upregulation of vimentin and $\mathrm{N}$-cadherin to promote epithelial-mesenchymal transition (EMT) in breast cancer cells (34). However, the effect of LAPTM4B on EMT in bladder cancer requires further investigation. LAPTM4B was also associated with tumor proliferation, angiogenesis, and poor prognosis in patients with glioblastoma, which could be used as a potential novel prognostic marker of glioblastoma to improve its treatment (35). Ethylglyoxal bisthiosemicarbazon, a specific inhibitor of LAPTM4B, was found to have effective antitumor activity in HCC (36).

In conclusion, the role of LAPTM4B was identified in bladder cancer progression using a range of different experiments.

\section{Acknowledgements}

Not applicable.

\section{Funding}

No funding was received.

\section{Availability of data and materials}

The datasets used and/or analyzed during the present study are available from the corresponding author upon reasonable request.

\section{Authors' contributions}

YY, YF, GY, and YD conceived the study, performed the molecular biology and in vivo experiments, performed the statistical analysis and drafted the manuscript. YY and YD confirm the authenticity of all the raw data. All authors read and approved the final manuscript.

\section{Ethics approval and consent to participate}

All procedures performed in the current study were approved by the Ethics Committee of Liaocheng People's Hospital (Shandong, China). Written informed consent was provided by all the patients or their families.

\section{Patient consent for publication}

Not applicable.

\section{Competing interests}

The authors declare that they have no competing interests.

\section{References}

1. Yao X, Li D, Xiong DM, Li L, Jiang R and Chen JX: A novel role of ribonuclease inhibitor in regulation of epithelial-tomesenchymal transition and ILK signaling pathway in bladder cancer cells. Cell Tissue Res 353: 409-423, 2013.

2. Yang Y, Deng S, Zeng Q, Hu W and Chen T: Highly stable selenadiazole derivatives induce bladder cancer cell apoptosis and inhibit cell migration and invasion through the activation of ROS-mediated signaling pathways. Dalton Trans 45: 18465-18475, 2016.

3. Soria F, Krabbe LM, Todenhöfer T, Dobruch J, Mitra AP, Inman BA, Gust KM, Lotan Y and Shariat SF: Molecular markers in bladder cancer. World J Urol 37: 31-40, 2019.

4. Ferlay J, Colombet M, Soerjomataram I, Mathers C, Parkin DM, Piñeros M, Znaor A and Bray F: Estimating the global cancer incidence and mortality in 2018: GLOBOCAN sources and methods. Int J Cancer 144: 1941-1953, 2019.

5. Radosavljevic V and Belojevic G: Shortcomings in bladder cancer etiology research and a model for its prevention. Tumori 100: 1-8, 2014.

6. Jordan EJ and Iyer G: Targeted therapy in advanced bladder cancer: What have we learned? Urol Clin North Am 42: 253-262, ix, 2015.

7. Pan CX, Zhang H, Tepper CG, Lin TY, Davis RR, Keck J, Ghosh PM, Gill P, Airhart S, Bult C, et al: Development and characterization of bladder cancer patient-derived xenografts for molecularly guided targeted therapy. PLoS One 10: e0134346, 2015.

8. Hou T, Zhou L, Wang L, Kazobinka G, Zhang X and Chen Z: CLCA4 inhibits bladder cancer cell proliferation, migration, and invasion by suppressing the PI3K/AKT pathway. Oncotarget 8: 93001-93013, 2017.

9. Ciccarese C, Massari F, Blanca A, Tortora G, Montironi R, Cheng L, Scarpelli M, Raspollini MR, Vau N, Fonseca J and Lopez-Beltran A: Tp53 and its potential therapeutic role as a target in bladder cancer. Expert Opin Ther Targets 21: 401-414, 2017. 
10. Blom T, Li S, Dichlberger A, Bäck N, Kim YA Loizides-Mangold U, Riezman H, Bittman R and Ikonen E: LAPTM4B facilitates late endosomal ceramide export to control cell death pathways. Nat Chem Biol 11: 799-806, 2015.

11. Zhang H, Qi S, Zhang T, Wang A, Liu R, Guo J, Wang Y and $\mathrm{Xu}$ Y: miR-188-5p inhibits tumour growth and metastasis in prostate cancer by repressing LAPTM4B expression. Oncotarget 6 : 6092-6104, 2015.

12. Li Y, Zhang Q, Tian R, Wang Q, Zhao JJ, Iglehart JD, Wang ZC and Richardson AL: Lysosomal transmembrane protein LAPTM4B promotes autophagy and tolerance to metabolic stress in cancer cells. Cancer Res 71: 7481-7489, 2011.

13. Zhou K, Dichlberger A, Martinez-Seara H, Nyholm TKM, Li S, Kim YA, Vattulainen I, Ikonen E and Blom T: A ceramide-regulated element in the late endosomal protein LAPTM4B controls amino acid transporter interaction. ACS Cent Sci 4: 548-558, 2018.

14. Meng Y, Wang L, Chen D, Chang Y, Zhang M, Xu JJ, Zhou R and Zhang QY: LAPTM4B: An oncogene in various solid tumors and its functions. Oncogene 35: 6359-6365, 2016

15. Yang H, Xiong F, Qi R, Liu Z, Lin M, Rui J, Su J and Zhou R: LAPTM4B-35 is a novel prognostic factor of hepatocellular carcinoma. J Surg Oncol 101: 363-369, 2010.

16. Xiao M, Jia S, Wang H, Wang J, Huang Y and Li Z: Overexpression of LAPTM4B: An independent prognostic marker in breast cancer. J Cancer Res Clin Oncol 139: 661-667, 2013.

17. Zhang H, Tian B, Yu H, Yao H and Gao Z: LAPTM4B-35 protein as a potential therapeutic target in gastric cancer. Tumour Biol 35: 12737-12742, 2014

18. Lebovitz CB, Robertson AG, Goya R, Jones SJ, Morin RD, Marra MA and Gorski SM: Cross-cancer profiling of molecular alterations within the human autophagy interaction network. Autophagy 11: 1668-1687, 2015.

19. Wang L, Meng Y, Xu JJ and Zhang QY: The transcription factor AP4 promotes oncogenic phenotypes and cisplatin resistance by regulating LAPTM4B expression. Mol Cancer Res 16: 857-868, 2018.

20. Tian M, Chen Y, Tian D, Qiao X, Ma Z and Li J: Beclin1 antagonizes LAPTM4B-mediated EGFR overactivation in gastric cancer cells. Gene 626: 48-53, 2017.

21. Livak KJ and Schmittgen TD: Analysis of relative gene expression data using real-time quantitative PCR and the 2(-Delta Delta C(T)) Method. Methods 25: 402-408, 2001.

22. Yang Y, Zhao Z, Xie C and Zhao Y: Dual-targeting liposome modified by glutamic hexapeptide and folic acid for bone metastatic breast cancer. Chem Phys Lipids 228: 104882, 2020.

23. Mahdavifar N, Ghoncheh M, Pakzad R, Momenimovahed Z and Salehiniya H: Epidemiology, incidence and mortality of bladder cancer and their relationship with the development index in the world. Asian Pac J Cancer Prev 17: 381-386, 2016.

24. Jung HK, Kim S, Park RW, Park JY, Kim IS and Lee B: Bladder tumor-targeted delivery of pro-apoptotic peptide for cancer therapy. J Control Release 235: 259-267, 2016.
25. Hussain SA, Palmer DH, Syn WK, Sacco JJ, Greensmith RMD, Elmetwali T, Aachi V, Lloyd BH, Jithesh PV, Arrand J, et al: Gene expression profiling in bladder cancer identifies potential therapeutic targets. Int J Oncol 50: 1147-1159, 2017.

26. Chen MW, Wei XD, Shi X, Lu L, Zhang G, Huang Y and Hou J: LncRNA HIF1A-AS2 accelerates malignant phenotypes of renal carcinoma by modulating miR-30a-5p/SOX4 axis as a ceRNA. Cancer Biol Med 18: 587-603, 2021.

27. Chen M, Zhuang C, Liu Y, Li J, Dai F, Xia M, Zhan Y, Lin J, Chen Z, He A, et al: Tetracycline-inducible shRNA targeting antisense long non-coding RNA HIF1A-AS2 represses the malignant phenotypes of bladder cancer. Cancer Lett 376: 155-164, 2016

28. Sun Z, Niu S, Xu F, Zhao W, Ma R and Chen M: CircAMOTL1 promotes tumorigenesis through miR-526b/SIK2 axis in cervical cancer. Front Cell Dev Biol 8: 568190, 2020.

29. Li S, Wang L, Meng Y, Chang Y, Xu J and Zhang Q: Increased levels of LAPTM4B, VEGF and survivin are correlated with tumor progression and poor prognosis in breast cancer patients. Oncotarget 8: 41282-41293, 2017.

30. Li L, Shan Y, Yang H, Zhang S, Lin M, Zhu P, Chen XY, Yi J, McNutt MA, Shao GZ and Zhou RL: Upregulation of LAPTM4B-35 promotes malignant transformation and tumorigenesis in L02 human liver cell line. Anat Rec (Hoboken) 294: 1135-1142, 2011.

31. Li Y, Iglehart JD, Richardson AL and Wang ZC: The amplified cancer gene LAPTM4B promotes tumor growth and tolerance to stress through the induction of autophagy. Autophagy 8: 273-274, 2012.

32. Meng Y, Wang L, Xu J and Zhang Q: AP4 positively regulates LAPTM4B to promote hepatocellular carcinoma growth and metastasis, while reducing chemotherapy sensitivity. Mol Oncol 12: 373-390, 2018

33. Meng F, Chen X, Song H and Lou G: LAPTM4B down regulation inhibits the proliferation, invasion and angiogenesis of HeLa cells in vitro. Cell Physiol Biochem 37: 890-900, 2015.

34. Xiao M, Yang S, Meng F, Qin Y, Yang Y, Jia S, Cai X, Li C, Huang Y and Ning X: LAPTM4B predicts axillary lymph node metastasis in breast cancer and promotes breast cancer cell aggressiveness in vitro. Cell Physiol Biochem 41: 1072-1082, 2017.

35. Dong X, Tamura K, Kobayashi D, Ando N, Sumita K and Maehara T: LAPTM4B-35 is a novel prognostic factor for glioblastoma. J Neurooncol 132: 295-303, 2017.

36. Li M,Zhou R, Shan Y, Li L, Wang L and Liu G: Targeting a novel cancer-driving protein (LAPTM4B-35) by a small molecule (ETS) to inhibit cancer growth and metastasis. Oncotarget 7: 58531-58542, 2016

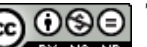

This work is licensed under a Creative Commons Attribution-NonCommercial-NoDerivatives 4.0 International (CC BY-NC-ND 4.0) License. 